\title{
Study on complexity and uncertainty perception and solution strategies for the time/cost trade-off problem
}

\begin{abstract}
In this paper, the Discrete Time/Cost Tradeoff Problem (DTCTP) is revisited in light of a student experiment. Two solution strategies are distilled from data of 444 participants and are structured by means of five building blocks, namely focus, activity criticality, ranking, intensity and action. The impact of complexity and uncertainty on the cost objective is quantified in a large computational experiment. Specific attention is allocated to the influence of the actual and perceived complexity and uncertainty and the cost repercussions when reality and perception do not coincide.
\end{abstract}

Keywords: Project Scheduling Game, Simulation, Complexity, Uncertainty

Cite as: Wauters, M., and Vanhoucke, M., 2016, "Study on complexity and uncertainty perception and solution strategies for the time/cost trade-off problem”, Project Management Journal, 47(4), 2950 .

\section{Introduction}

Time/cost trade-offs in project scheduling find their roots in the Critical Path Method (CPM), developed at the duPont company and at Remington Rand Univac (Kelley and Walker (1959), Walker and Sawyer (1959) and Kelley (1961)). CPM is a project scheduling technique to analyze and represent the tasks involved in completing a given project. Although this method does not explicitly take resource requirements into account, it assumes that the cost of an activity is a function of its duration. As the duration of an activity is decreased, its associated costs will rise, since more resources need to be allocated to that activity. Initial research efforts on the time/cost trade-off problem focused on the continuous case and can be found in standard texts such as Elmaghraby (1977) and Moder et al. (1983). Several techniques were used to solve this type of problem (Robinson (1975), Hindelang and Muth (1979), Phillips and Dessouky (1977) and Meyer and Shaffer (1965)). An overview of the literature until the mid nineties is given by De et al. (1995). We will cover the contributions related to the time/cost trade-off problem from the mid nineties onwards. The Discrete Time/Cost Trade-off Problem (DTCTP), shown to be NP-hard by De et al. (1997), was solved exactly by Demeulemeester et al. (1996). In this paper, the authors present two approaches based on dynamic programming for reaching the optimal solution of the three objective functions of the DTCTP. Three possible variants of the time/cost trade-off problem can be identified. Scheduling project activities with the goal of minimizing the total project costs while meeting an 
imposed deadline is known as the deadline problem (DTCTP-D). The budget problem specifies a limit on the budget (DTCTP-B). The objective is then to minimize the duration of the project. Finally, the third objective deals with generating a complete and efficient time/cost profile. Demeulemeester et al. (1998) improved the computational results for solving the DTCTP optimally. This is done using a branch-and-bound procedure that calculates lower bounds by convex piecewise linear underestimations of the time/cost trade-off curves of the activities. This contribution is of special relevance to this paper since it will be used to provide an optimal solution for the data instances of the computational experiment.

The last decade, two new research avenues on the time/cost trade-off problem were examined. The first new direction is the extension of the (D)TCTP, while the second direction focuses on the inclusion of stochastic characteristics to the (D)TCTP. A brief overview of the key publications belonging to each avenue, along with their contribution, is provided in Table 1.

\section{INSERT TABLE I HERE}

The contribution of this paper to the existing body of literature is threefold. First of all, two solution strategies of students participating in a project management business game, the Project Scheduling Game (PSG), are distilled. These solution strategies are a combination of five building blocks, namely focus, activity criticality, ranking, intensity and action. Secondly, we take two contextual factors, namely complexity and uncertainty, into account. While the first contribution makes use of real-life data, experiments are constrained by the fact that classroom sessions need to be held in order to gather additional data. The final contribution overcomes this problem by testing the derived solution strategies on computer-generated project networks. In the remainder of this section, we will elaborate on these contributions from a literature point of view.

Business games Business games have a long history within an educational context. Early research focused on the internal validity through assessing advantages and disadvantages of simulations versus other pedagogies (Schumann et al. (1997)). Later on, the validity of top management games was confirmed by Wolfe (1997). The most-cited advantages of the use of business games are their high degree of realism, a broader learning environment, competition between players, as well as soft skills such as communication skills, group behaviour and organization skills (Saunders (1997) and Faria (2001)). On top of this, business games craft personal experiences by challenging participants on an intellectual and behavioural level and hence fall within the nominator experiential learning (Kolb (1984)). Parente et al. (2012) argue in favour of business games by stating that real-life experience imposes limitations since there is no opportunity to experience the full range of 
possibilities and skill development. Business games have been applied to simulate business and operations management in the electronics industry (Haapasalo and Hyvönen (2001)), to teach business ethics (Schumann et al. (1997)), to develop entrepreneurial skills (Stumpf et al. (1991)) and to enhance systems thinking and business process redesign (Van Ackere et al. (1993)).

Complexity and uncertainty The papers of Table 1 all focus on what Pollack (2007) describes as the hard paradigm, which is commonly associated with quantitative techniques and deductive reasoning. However, the author identifies research streams that suggest an increasing acceptance of the soft paradigm, which focuses on qualitative techniques that emphasize contextual factors and relevance. Examples of soft paradigm publications can be found in Turner and Müller (2005), Ojiako et al. (2014), Green (2004) and Yang et al. (2011) and the reader is referred to the relationship school and behavioural school of Söderlund (2011) for a literature review on soft paradigm aspects. More and more, researchers are calling for a broader view of project management (Hanisch and Wald (2011)), an increased alignment of research and practice (Hall (2012)) or the inclusion of contextual factors (Crawford et al. (2006)). With regard to the latter point, Maylor et al. (2008) argue that onesize-fits-all approaches are inconsistent with the contextual diversity managers are confronted with. In this paper, we take two contextual factors, namely complexity and uncertainty, into account by means of data from students participating in a business game.

The choice for complexity and uncertainty is inspired by two reasons. Firstly, Howell et al. (2010) noted that uncertainty was easily the most dominant theme, with complexity ranking second. This confirmed the findings of Shenhar (2001) who discovered an emergence of uncertainty and complexity based on a review of the classical as well as the more recent literature. It is worth noting that the works dealing with stochastic characteristics in Table 1 revolve around uncertainty. Additionally, the works of Thomas and Mengel (2008) and Hanisch and Wald (2011) explicitly recognize complexity and uncertainty as crucial (contextual) factors. Secondly, integrating contextual factors can be regarded as a response to areas for future research. Hanisch and Wald (2011) argued that the influence of complexity on the project outcome needs to be studied, while Maylor et al. (2008) wondered whether a quantification of complexity was feasible. While research on uncertainty has witnessed a spike in interest from academics, it is still among the top challenges for future research (Hall (2012)).

Simulation Complexity can be defined from a hard paradigm perspective (see the complexity measures of Pascoe (1966), Mastor (1970), Bein et al. (1992) and De Reyck and Herroelen (1996)) or include soft paradigm aspects (such as organizational complexity (Wolfe (1996)) or socio-political complexity (Geraldi et al. (2011)). 
In this work, we focus on structural complexity and more specifically on system size (Sommer and Loch (2004)). Apart from a large body of work that supports this stance (Dvir et al. (2006), Geraldi and Adlbrecht (2007), Müller and Turner (2007)), the main rationale for focusing on system size can be found in the final contribution this paper makes. Once the student files are analyzed and turned into solution strategies, these strategies are applied to computer-generated project networks, for which multiple settings are changed. In order to do this, a more technical definition of complexity (and uncertainty) is required. Specific attention will be allocated to the discrepancy between the actual complexity and uncertainty and how these contextual factors are perceived. Individuals perceive reality in their own way (Jaafari (2003)), implying that complexity and uncertainty are also in the eye of the beholder (Nutt (1998), Vidal and Marle (2008), Osman (2010), Ojiako et al. (2014)). Simulations aid decision makers in anticipating and quantifying the effects of actions and events (Fang and Marle (2012)) and allow us to generate a wide spectrum of outcomes.

The outline of the paper is as follows. In section 2, a general overview of the Project Scheduling Game is given. Section 3 focuses on the data collection phase. The building blocks of the solution strategies, the link to the student data of section 2 and how the solutions are evaluated are discussed in section 4. An illustrative example is provided to demonstrate the 5 components that make up a solution strategy. Section 5 introduces the general framework that forms the foundation for the solution strategies and introduces the time-based and cost-based solution strategy. Section 6 includes details about the test design. Parameter settings are divided depending on whether they are project-specific or whether they relate to the complexity and uncertainty dimension. The results of the solution strategies are discussed in section 6.2 , where a distinction is made between the general performance, the performance in case of judgement errors and an investigation of a varying degree of level of effort. Finally, a discussion of the results and general conclusions can be found in section 7 .

\section{Game description}

Crowston and Thompson (1967) were among the first authors to stress the importance of the interaction between the planning, scheduling and control phase of a project. The focus of the Project Scheduling Game, presented by Vanhoucke et al. (2005), lies in the scheduling and control phases of the project life cycle. More precisely, it is the aim of the player to follow an iterative approach, known as reactive scheduling, that compares the project baseline schedule with the current project performance (simulated during the execution phase) in order to control the project and take corrective actions in case the project objective is in danger. The game consists of several phases which require periodic input from the game player. An overview of the game process is given in figure 1 . 


\section{INSERT FIGURE I HERE}

First of all, the project network, along with a baseline schedule and other input data for the game such as the trade-off details are proposed by the course teacher. The baseline schedule ends at time $\mathrm{T}$. In order to acquaint students with uncertainty, unexpected events occur. A new deadline, $\delta_{n}<T$, is imposed by the client along with a penalty cost for every day the deadline is exceeded. Therefore, the PSG imposes a soft deadline that need not be met. However, late project delivery is discouraged by means of a penalty that is incurred for every day the project finishes after $\delta_{n}$. These changes require an update of the baseline schedule which is the task of the player of the game. The update process of the baseline schedule boils down to a new trade-off selection for a number of activities. New trade-offs lead to a shortened or prolonged duration of an activity and lie at the heart of the CPM. Second, the project is divided into multiple decision moments. The game then simulates periodic project progress in which uncertain events might occur. Changes to the original activity durations lead to deviations from the initial baseline schedule and endanger the project objective. For every decision moment, the player has to evaluate periodic review reports and rebaseline the unfinished activities of the project schedule in order to bring the project back on track. This process of rescheduling, taking a decision and assessing the new information is repeated until the final decision moment is reached. Third, after a predefined number of decision moments, the game reports the final project status in terms of the total project duration and cost. At this point, feedback is given by the course teacher regarding the main learning objectives of the PSG.

\section{Data collection}

The PSG is taught to management and engineering students at 2 universities (Ghent University (UGent, Belgium) and University College of London (UCL, United Kingdom)) and 2 business schools (Vlerick Business School (VBS, Belgium) and the EDHEC business school (France). 444 data points were collected, among which 176 business engineering students (UGent), 203 civil engineering students (UGent), 36 civil engineering students (UCL), 5 management science students (UCL) and 24 MBA students at the VBS and EDHEC school. Most of these students do not have previous working knowledge. It is worth remarking that the students business engineering and most of the civil engineering students hail from the same university (UGent).

A distinction can be made between data captured during game progress and final results. All the data captured during game progress is saved in a log file. For every student playing the PSG, a log file is available. These log files store commands students execute. The most important commands are the change of an activity's trade-off option and taking a decision, implying a move to the next decision moment. Taking a decision executes the new trade-off settings for the activities and simulates project progress for the next decision moment. Examination of 
the commands leading to intermediate and final solutions permits an identification and classification of solution strategies taken by the students. For instance, by executing the commands stored in the log files chronologically, it is possible to know whether a student made a change to a critical or non-critical activity and what that change consisted of. The final time and cost solution for every student is only available at the end of the game. Table II summarizes the results of the different student groups. The average deviation compared to the global minimum cost point is less than $2 \%$ for all student groups. The best solution of the student groups displays only a very small deviation from the best solution possible (less than 1\%). The best overall solution was found by a business engineering student, whereas on average, the management science students of UCL report the best average score. They also achieve the smallest standard deviation in cost and the lowest maximum cost deviation. It is worth remarking that the group of management science students at UCL only comprises 5 people. Welch's t-test was applied to find out whether the cost deviations between the groups differ significantly. The only statistically significant difference $(\mathrm{p}<0.05)$ is found for the students Civil Engineering at UGent and the students Civil Engineering at UCL.

\section{INSERT TABLE II HERE}

\section{Data structuring}

Based on the aggregated data, the log files and time/cost deviations, it is necessary to transform the data into information by finding a certain structure according to which students play the PSG. This is accomplished by looking for recurring data patterns. A data pattern that corresponds with a certain class of behaviour, exhibited by many students will be called a solution strategy. Section 4.1 expands on the 5 building blocks that form one solution strategy. The following section explains the link between the solution strategy components and the student data of section 3. Section 4.3 presents details about the performance measures that will be used to evaluate the strategies. Section 4.4 provides an illustrative example and shows the different steps going from activity selection to applying a trade-off change.

\subsection{Solution strategy components}

From the data collection phase and through many discussions with the students at the educational institutions, we learned that very few students approach the PSG without any underlying logic. It is possible to discern 5 building blocks that are in line with when and how students select a new mode for the different activities. The 5 components that characterize a solution strategy are focus, activity criticality, ranking, intensity and action. It is worth noting that the first 4 elements are related to selecting a set of activities whereas the final element, action, determines how the trade-offs of the set of activities will be altered. This corresponds with the three general building blocks of heuristics (Gigerenzer and Gaissmaier (2011)). Search rules specify the direction of the 
search space and are accounted for by focus, activity criticality, ranking and intensity. Stopping rules determine when the search process ends and is governed by the time limit of the PSG. Decision rules elaborate on how the final decision is reached. This is done by the fifth component, namely action.

At the start of a decision moment, every activity that has not started is subject to a possible change. Out of this group of activities, focus, activity criticality, ranking and intensity perform a stepwise selection of a subset of activities. The process of stepwise selection can be described as follows:

- Focus: specifies the length of the time window during which actions will be taken. All activities that start or are still in progress during this time window are selected. The focus is expressed as a percentage of the number of decision periods that are taken into account. The focus can vary from a local to a global orientation. A local orientation is characterized by a narrow time window because the number of decision periods taken into account is small. At the other end of the spectrum is a global orientation, which uses a wide time window. In this case, many activities will be subject to a possible trade-off change.

- Activity criticality: the subset of activities that start or are in progress during the time window specified by the focus can be further refined based on whether these activities are critical or non-critical at the current decision moment. If both critical and non-critical activities are taken into consideration, the subset of activities before this phase equals the subset at the end of the phase.

- Ranking: the elements of the subset of activities are ranked based on the value of a priority rule. Within the context of human decision-making, priority rules are easy to apply and in line with techniques that are used to give priority to certain activities. A tight match could be witnessed between the followed solution strategy and the selected priority rule. For instance, students who thought that the minimum cost solution would lie in the neighbourhood of the deadline would adopt a more time-based strategy and select a priority rule that takes into account activity durations. This selection step does not reduce the subset of activities but accords a ranking to the activities. These rankings serve as input for the intensity phase. The priority rules used by the solution strategies are the Greatest Rank Positional Weight (GRPW), Maximum Slack (MAXSLK) and Average Most Expensive Activity (the activity cost divided by its duration) rules.

- Intensity: given the fact that students have a limited amount of time to take decisions, it is crucial to focus on the most important activities. Intensity further selects activities by determining a cut-off point for the ranked subset that resulted from the previous phase. A percentage between $0 \%$ and $100 \%$ of the number of remaining activities of the ranked subset is used as a value for the intensity. This percentage is multiplied by the number of elements that are present in the ranked subset.

This subset then serves as input for the action phase, where the trade-offs of activities that are elements of the 
subset may be changed.

- Action: an action is defined as a move on the trade-offs of an activity that may potentially change an activity's cost and associated duration. This need not be the case since a lot of students check whether the action leads to an immediate cost decrease or not. If there is no improvement, it is possible that the activity's cost and duration is reverted. Actions can go from simple to more advanced operations. An overview of the type of actions and an accompanying description is given in Table III.

\section{INSERT TABLE III HERE}

The rationale for the different refinement phases leads back to the nature of the PSG, where students only have a limited amount of time to make changes and advance to the next decision moment. Hence, it is necessary to focus on the activities that are most important. In order to clarify the building blocks of the solution strategies, the stepwise selection and action will be illustrated using a straightforward example.

\subsection{Link to the student data}

The previous section outlined the different building blocks of a solution strategy. The aim of this section is to connect the 5 components (focus, activity criticality, ranking, intensity and action) to the student data of section 3. The link between these two sections results from two aspects, namely data analysis and the feedback sessions with the participants of the Project Scheduling Game. Most of the time, these aspects go hand in hand. For instance, many of the conversations revealed that students first select a number of activities to which a trade-off change can be made. Further questioning led to the formalization of this selection and to the inception of focus and intensity.

Focus The values for the focus and intensity could be retrieved from the log files of the students. We witnessed that a wide focus range is used by the students, which explains why low and high numbers for the focus are used by the solution strategies.

Activity criticality Activity criticality is the second of the five building blocks that make up a solution strategy. It is logical that both critical and non-critical activities are changed.

Ranking Arguably, the student feedback proved most valuable in identifying the priority rules for the ranking phase that were used most often, especially since these are much harder to keep track of in the log files.

Intensity The process for translating intensity from the student data to the solution strategies was slightly different. Intensity, as defined in section 4.1 , can reach any value in the interval $[0,100]$. A careful trade-off needs to be made between a sufficient data representation and having as few values for the intensity setting as possible. In theory, it would be possible to incorporate all possible values the intensity can achieve in the solution strategies. However, this entails that a huge number of branches need to be created to test which value 
will be applied under which circumstances. Such a situation reflects the data in an extremely accurate fashion but is no longer feasible for the computational experiment. As a rule of thumb, the fewer different values that represent the data of the students' adopted intensity well, the better. Feedback from the students taught us that selection of a number of activities typically occurred through a rule-of-thumb, such as " 1 out of 4 activities" will be retained. There are two principal reasons why a limited number of values for the intensity were embedded in the solution strategies. First of all, from a cognitive point of view, it is better if the different values are almost equally spaced in the interval that ranges from 0 to 100 . Secondly, while there are only a limited number of different values, the data is represented in a sufficiently accurate way.

Action The final element, the actions, once again resulted from the feedback and the $\log$ files. For this dimension, it is easier to find in the data which actions were followed, with one notable exception. An enumeration of different trade-off options can easily be confused with sequential swaps. The data showed that participants of the game make frequent use of swapping trade-off options, which explains the frequent inclusion of this action in the solution strategies.

\subsection{Evaluation}

The solution strategy components of the previous section were derived using the data accessible from the log files. As mentioned in section 3, the second type of data, the results, are gathered at the end of the game. In order to rate the quality of the student solutions, represented by the derived solution strategies, it is necessary to define performance measures. The proposed measures capture 2 dimensions of a final solution, namely cost and level of effort. Every dimension can be measured using a specific metric, which is outlined below and depicted in figure II. The $\mathrm{x}$-axis of figure II represents the deviation from the deadline in absolute numbers, whereas the $\mathrm{y}$ axis displays the total costs. The curve shows the efficient time/cost profile. The time value of the minimum cost solution across the entire efficient time/cost profile is denoted by $\mathrm{t}^{*}$. Finally, the dot stands for the solution of a student at the end of the game.

- Cost performance: the cost deviation is measured using the global cost deviation. This deviation compares the project cost of the solution strategy (the dot) to the solution that yields the minimum cost across all possible time points of the complete time/cost profile (the cost or $y$-value at time $\mathrm{t}^{*}$ ). The global cost deviation is expressed as a percentage deviation:

$$
\Delta \operatorname{cost}_{\text {global }}=\frac{c^{s}-c_{t^{*}}^{*}}{c^{s}}
$$

In this calculation, $c^{s}$ stands for the cost of the solution strategy and $c_{t^{*}}{ }^{*}$ denotes the cost of the efficient 
time/cost profile at time $t^{*}$. It is possible to break down the global cost deviation into 2 constituent parts, namely activity costs and penalty costs. If a project finishes later than the specified deadline, it incurs a penalty cost. Hence, by looking at the penalty cost, we implicitly derive some information as well.

- Level of effort: captures how much effort it takes to reach a solution, which results from one of the solution strategies. It is worth noting that the level of effort is a function of focus, activity criticality and intensity. As the focus increases, the amount of potential activities that are changed rises and consequently, the level of effort increases as well. This dimension aims to establish the link between solution quality and the amount of work that was performed to reach that solution. One unit of effort corresponds with one trade-off option that is considered for a change. For instance, in order to determine the minimum cost slope, a number of trade-off options are considered. Each of those trade-offs augments the level of effort by 1 unit.

\section{INSERT FIGURE II HERE}

\subsection{Illustrative example}

Figure III(a) represents the Activity on the Node (AoN) notation of an example network. We note that this example merely serves as an illustration: the networks of the computational study count more activities and different trade-off options. In this example, there are 7 activities in total. The possible durations of the trade-off options for every activity are indicated above each node. The associated costs of the trade-offs are listed under each node. The currently selected trade-off is bolded. For instance, for activity 2, the currently selected trade-off has a duration equal to 2 at a cost of $€ 100$. Figure III(b) depicts the earliest start Gantt-chart, taking into account the precedence relations between the activities. Critical activities (activities 1, 3, 4,5 and 7) are highlighted in grey, whereas non-critical activities are indicated by the non-coloured bars (activities 2 and 6). There are also 3 different decision moments (DM). In this example, it is possible to make changes to a set of activities at time points 0,5 and 10 respectively. The total duration of the project equals 16 days. We assume that a decision needs to be made at time point 0 and that a deadline of 13 days is present. If the project duration exceeds the deadline, a penalty cost of $€ 100 /$ day is incurred. Hence, we are at the beginning of the project. Let E denote the set of eligible activities. Eligible activities are defined as activities for which the currently selected trade-off will be changed. Hence, at the start of the project, the set $E$ consists of every activity present in the network:

$$
\mathrm{E}=\{1,2,3,4,5,6,7\}
$$


The example settings for the 5 components (focus, activity criticality, ranking, intensity and action) are listed in Table IV. The focus is assumed to be equal to 2 decision moments $(0.67 * 3$, the total number of decision moments). From that set of activities, only the critical activities will be retained. These will then be sorted according to the Most Expensive Activity priority rule. From the ranked subset, only the first $67 \%$ will be withheld. Finally, those remaining activities will be crashed by applying a swap operator.

\section{INSERT TABLE IV HERE}

- Focus: in this example, a focus of 2 decision moments is used. Given the fact that the current decision moment is equal to 1 , the time window for the activities that will be retained is equal to:

$$
\text { Time window }=[1,1+2[
$$

Hence, activities that start later than decision moment 3 (after time point 10) will no longer be considered.

$$
\mathrm{E}=\{1,2,3,4\}
$$

- Activity criticality: only critical activities will be taken into consideration. This implies that activity 2 will be removed from $\mathrm{E}$.

$$
\mathrm{E}=\{1,3,4\}
$$

- Ranking: cost is the most important objective for this example network. Hence, the priority rule Most Expensive Activity will be used. Activity 1 has a cost of $€ 50$, activity 3 a cost of $€ 60$ and activity 4 costs $€ 40$. Consequently, the activities in $\mathrm{E}$ are reordered as follows:

$$
\mathrm{E}=\{3,1,4\}
$$

- Intensity: further refinements can be made based on the intensity. In this example, an intensity value of 0.67 will be used. This means that only $2(0.67 * 3$ activities $)$ activities will be left. Because of the ranking in the previous phase, the first two activities will be selected.

$$
\mathrm{E}=\{3,1\}
$$

The 5 building blocks have gone from a set where all activities could be changed to a situation where only 2 activities are left. In the final phase, an action will be applied to those activities to change their selected tradeoff:

- Action: activities 3 and 1 will be crashed by selecting the neighbouring trade-off option. This implies that the duration of activity 3 will become 3 time units, with a cost of $€ 120$. Activity 1 will now take 4 days to complete at a cost of $€ 80$. This leads to the Gantt-chart in figure IV, which will be the initial situation for the next decision moment (decision moment 2). The Gantt-chart indicates that the critical path has changed and now consists of activities 1, 4,6 and 7. The total duration of the project has decreased from 16 to 14 days. The project's total cost has decreased from $€ 690(€ 390+3$ days $* € 100)$ 
to $€ 580(€ 480+1$ day $* € 100)$.

\section{INSERT FIGURE IV HERE}

In order to demonstrate the trade-off between solution quality and Level Of Effort, 4 solution strategies are applied to the toy example described in the paragraphs above. The first strategy consists of doing nothing, yielding a total cost of $€ 690$. Strategy 1 employs a focus and intensity of 0.33 , strategy 2 assumes a focus and intensity of 0.66 and strategy 3 adopts a maximal focus and intensity (=1). The upper part of Table V shows which activities were changed or considered for a change. The lower part displays the project's final outcome and compares it to a scenario where no action is taken. While there is an improvement in cost between doing nothing and strategies 1 and strategies 1 and strategies 2, there is no advantage in increasing the focus and intensity beyond 0.66 . Hence, it is shown how an increasing focus and intensity improves the cost objective until a point is reached where further increases lead to a dramatic increase in Level Of Effort, to the same cost solution or both.

\section{INSERT TABLE V HERE}

\section{Strategic Framework}

The strategic framework contains information about the conditions in which the derived solution strategies operate. Two defining criteria are determined, namely complexity and uncertainty. The general framework of the solution strategies, as well as complexity and uncertainty appraisal, are the subject of section 5.1, in which the interplay of these dimensions with the solution strategies will be clarified. Section 5.2 lists the 2 proposed solution strategies. One of these strategies will concentrate on time while the other strategy adopts a cost-based point of view.

\subsection{General framework}

Section 4 described the 5 building blocks of a solution strategy: focus, activity criticality, ranking, intensity and applying an action. These elements will be used to construct the strategies that were derived based on the data collected in the log files and based on the discussions with the students after finishing the game. However, the proposed strategies take 2 important criteria into account, namely complexity and uncertainty. A crucial element throughout this paper is the difference between the real and the perceived complexity or uncertainty. People make decisions based on the perceived complexity or uncertainty without knowing their real value. Hence, it is possible that judgement errors, in which the threshold value differs from the real value, occur. If the uncertainty or complexity estimate exceeds a threshold value, the outcome for that dimension will be judged high. Otherwise, that dimension will be judged low. Details on these thresholds will be provided in section 6.1. The 
outcome of the judgement of the complexity and uncertainty will steer the logic of the solution strategies into a different direction. This implies that different settings for the stepwise activity selection (focus, activity criticality, ranking and intensity) and action phases may be applied. Complexity is measured by the average number of trade-offs of the different activities and is calculated as follows:

$$
C=\frac{\sum_{i=1}^{n} n r t o_{i}}{n}
$$

with $n$ denoting the total number of activities and $n r t o_{i}$ the number of trade-off options for activity $i$. Students often use the proportion of activities that were subject to a delay in previous decision periods as an indicator for future uncertainty. The outcome of the complexity and uncertainty criteria is a binary value: either a project is highly complex (or very uncertain) or not. The values for complexity and uncertainty are taken into account by all the solution strategies. The actual complexity and uncertainty is imposed by the decision maker. Individuals differ on how they judge complexity and uncertainty (Mintzberg et al. (1976), Bourgeois (1985)), which will be imitated in the computational experiment by incorporating different threshold values, leading to a different judgement of complexity and uncertainty.

The general framework of the solution strategies is given in figure V. At the start of the project, the complexity is analyzed. If the complexity is low and the project is about to start (the decision moment equals 0), a group of settings and actions labeled $\mathbf{A}$ is triggered. If some activities are finished already, a new estimate of the uncertainty is made. If uncertainty has shifted from low to high or high to low, the focus and intensity are adapted (B). If uncertainty is smaller than the threshold value $(\mathrm{U}<)$, it is checked whether the activity is critical. If this is the case, $\mathbf{C}$ will be triggered. In the alternative case, the settings and actions encompassed in $\mathbf{D}$ are executed. Finally, if uncertainty is high, a similar check with regard to the (non-)critical nature of an activity is performed. If the activity is critical and has only 1 predecessor, the procedure moves to the branch labeled $\mathbf{E}$. If the activity is non-critical, the actions and settings comprised in $\mathbf{F}$ will be activated. A similar but slightly more intricate pattern is executed in case the project's complexity exceeds the threshold value. When the project has just started, a couple of additional branches (G-I) are present. Furthermore, if a critical activity has more than 1 predecessor, a set of settings and actions will be applied as well. After applying a set of actions from one of the possible groups, a check is performed in order to determine whether the project has ended. If this is not the case, the project moves to the next decision moment. Otherwise, the output measures are calculated and the solution strategy has come to an end. This framework and its different branches will be used in the next section to structure the proposed solution strategies. 


\subsection{Proposed strategies}

Armed with the student data and the structure explained in section 5.1, it was possible to derive 2 solution strategies. The first strategy, treated in section 5.2.1, focuses on time and more specifically on reaching the imposed deadline $\delta_{n}$. The second strategy employs cost-saving measures at the expense of increased risk and is discussed in section 5.2.2. The level of effort was controlled using the focus and intensity parameters, which differ based on the level of complexity and uncertainty. This was necessary to ensure that the level of effort was equal across all settings for the computer experiment.

\subsubsection{Time strategy}

The goal of the time strategy is to approach the deadline as closely as possible. This solution strategy, abbreviated $\mathrm{SS}_{1}$, uses three specific mechanisms that employ a time-based focus. First of all, the Greatest Rank Positional Weight (GRPW) rule is invoked in several branches. This rule takes the duration of the activity under study and the durations of its immediate successors into account, thus capturing a small portion of the network structure. Secondly, a buffer mechanism is employed. The buffer is based on the Slack Duration Ratio (SDR) by Hazir et al. (2010) and implies that a minimum value for the ratio of an activity's slack to its duration should be maintained. Consequently, non-critical activities are protected against delays that could turn them into critical activities and delay the entire project. The buffer mechanism is invoked when the uncertainty is judged to be high. Finally, the protect deadline action is used to ensure that the project duration does not deviate too much from the imposed deadline. Protection of the deadline is done when the project is in progress (DM $\neq 0)$. It ensures that the deadline does not exceed student-specified bounds. If the project is slightly uncertain, the project duration should lie between $98 \%$ and $101 \%$ of the deadline. For highly uncertain projects, the lower bound on the deadline becomes $96 \%$. Because of the increased uncertainty, the delays will push the duration closer to the deadline, which explains why a smaller lower bound is chosen. The downward protection of $98 \%$ and $96 \%$ is not applied when the complexity is judged high. In this case, more effort is put into the examination of different trade-off options. However, the protection of $101 \%$ of the deadline is still in place in order to minimize penalty costs.

\subsubsection{Cost strategy}

The cost strategy, $\mathrm{SS}_{2}$, aims to minimize the sum of activity costs and the penalty cost. Contrary to the time strategy, taking risk will be an integral part of this solution strategy. This is done using 3 different mechanisms. First and foremost, the average most expensive activity rule is used a lot more compared to the time strategy, implying that the importance of costs versus the duration of an activity becomes more important. Secondly, the elitism criterion is used frequently. Only accepting better solutions can be done for simple and complex actions, 
for instance by selecting the best outcome of a minimum cost or maximum revenue slope. Third, consumption of slack plays a central role. Including this action has a double effect. On the one hand, costs will decrease because longer activity durations (at a lower cost) will be selected. On the other hand, because there is less slack in the project's schedule, the amount of risk increases. The logical result is that activity delays will have a larger impact on the schedule, thus increasing the penalty costs. Again, a distinction is made between judging projects to possess a low or high degree of complexity.

\section{INSERT TABLE VI HERE}

A summary of the principal differences between both solution strategies is given in Table VI. The reader is referred to Table VII for an exhaustive overview of the two proposed solution strategies. While this Table is mainly relevant for researchers who want to imitate our computational experiment with identical parameter settings, it can easily be skipped by the reader without losing the general overview of the theme of this paper. Table VII lists the settings of the solution strategy components for every branch. The letters of the respective branches correspond with those depicted in figure V. For focus and intensity, an additional distinction is made based on the actual complexity, which can be low (denoted $\mathrm{C}_{\mathrm{L}}$ ) or high (denoted $\mathrm{C}_{\mathrm{H}}$ ). For the ranking component, Avg MEA is the abbreviation for the average Most Expensive Activity priority rule, whereas Max SLK denotes the maximum slack priority rule. Finally, in the action column, MC represents the Minimum Cost while MR stands for the Maximum Revenue. The subscript elit refers to elitism, meaning that if an action leads to a cost deterioration, the action will be undone and the project reverts to the trade-offs before the action was applied. For a step-by-step procedure of the data generation phase and the settings of the solution strategies, the reader is referred to the Appendix.

\section{INSERT TABLE VII HERE}

\section{Computational Experiment}

The computer experiment aims to reproduce the behaviour exhibited by the students on a diverse set of generated projects. The goal of this section is not to compete with existing exact and (meta-)heuristic approaches but rather to discern the circumstances in which each solution strategy reaches the best results. In fact, large cost deviations illustrate the limitations of human decision makers and identify the need for more involved population-based optimization techniques. These more advanced techniques were discussed in the literature overview of section 1. The outline of this section is as follows. Section 6.1 provides details about the data generation process where a distinction is made between project-based parameter settings and settings related to the complexity and uncertainty. A baseline scenario is defined and will serve as the main vehicle to illustrate predominant relations for the different complexity and uncertainty combinations. Using this baseline 
scenario, the impact of judgement errors on the cost performance is studied. Finally, the effect of a varying level of effort is discussed.

\subsection{Data generation}

A distinction can be made between project-based parameter settings and settings related to the complexity and uncertainty. First of all, the project-based parameters will be discussed and the baseline scenario will be established. Afterwards, the settings related to complexity and uncertainty are divulged. All of these settings are summarised in Table VIII and discussed in the following paragraphs. Table VIII is illustrated on a specific example and combined with the settings of one of the solution strategies in the Appendix.

Project-based settings 100 project networks with 30 activities were generated using the RanGen2 generation engine (Vanhoucke et al. (2008)) for 9 values of the Serial/Parallel (SP) indicator, ranging from 0.1 to 0.9 in steps of 0.1. Although the SP indicator is named the I2 indicator in the paper by Vanhoucke et al. (2008), it is commonly referred to as the SP indicator in several simulation studies (e.g. Vanhoucke (2010)). The SP indicator measures a network's degree of closeness to a completely serial or parallel network (Tavares (1999)). The following project-based parameter concerns the nature of the generated trade-offs. These can be random, linear, convex or concave. Convex trade-offs entail steeply increasing costs as an activity's duration is crashed. The opposite observation holds for concave trade-offs. We only consider random trade-offs. Robustness checks were performed for linear, convex and concave trade-offs without leading to different results.

Imposing a deadline for the project is done after the instance is solved in an exact way, using the procedure of Demeulemeester et al. (1998). The exact solution method returns an efficient time/cost profile. Let $D_{\min }$ denote the minimum project duration and $D_{\max }$ denote the maximum project duration. The deadline, $\delta_{\mathrm{n}}$, is determined using the parameter $\theta$, as follows:

$$
\delta_{n}=D_{\min }+\theta *\left(D_{\max }-D_{\min }\right)
$$

3 levels for $\theta$ are suggested: $0.25,0.5$ and 0.75 . Finally, the penalty parameter determines how extremely exceeding the deadline is discouraged. A low penalty setting (€350 per day) and high penalty setting $(€ 3,500$ per day) are taken into consideration. The height of the penalty has a direct influence on the global cost deviation. If a solution is reached with only a small time deviation but the penalty is set to a high number, the global cost deviation will be much higher than a situation with a low penalty setting. The combination of a certain value for the deadline and penalty determines the location of the optimal cost on the efficient time/cost profile. Finally, for every possible mode, a combination of durations and costs needs to be generated. The number of modes will be specified in the complexity and uncertainty settings paragraph. Activity costs range 
from $€ 500$ to $€ 2,500$ with a maximum allowed interval of $€ 1,000$ between two modes of an activity. The minimum durations of an activity go from 10 to 20 with a maximum interval of 1 time unit.

\section{INSERT TABLE VIII HERE}

Baseline Scenario The baseline scenario is used as an instrument to identify the main effects of different combinations of the complexity and uncertainty parameter. The characteristics that closely resemble the PSG's properties were employed to construct this scenario. An exception is made for the penalty parameter, where both values were used.

Complexity and uncertainty settings Complexity refers to the average number of trade-offs of the different activities and was first introduced in section 5.1. There are two levels for the complexity of the generated projects. The activity modes of the projects are generated according to a triangular distribution with 1, 4 and 6 modes as the minimum, mode and maximum for projects with a low degree of complexity and 4, 7 and 9 modes for highly complex projects. There are two settings for the complexity threshold which determine if a project is judged to be complex or not. The first threshold setting is equal to 0 , thus indicating that every project will be judged complex. The other parameter value equals 10 and implies that every project will be judged lowly complex.

The second dimension is uncertainty, which consists of 3 elements, namely the uncertainty type, proportion and size. The delay type denotes the amount of positive and negative delays. A proportion of $90 \%$ positive delays (10\% negative delays) is put forward. Negative delays result in activities finishing earlier than planned and are included to reflect that uncertainty can also yield opportunities (Ward and Chapman (2003)). Consequently, the delay type penalizes or rewards risk takers who do not incorporate a lot of slack into their projects. Closely linked with the delay type is the percentage of activities subject to a delay. This is applied to move the project's execution closer to or further away from the baseline schedule. The percentage of activities subject to a delay is applied to all activities at the start of the project's execution. Hence, an activity can only be delayed once. If this percentage is low, few unanticipated delays will distort the decisions about the activity modes taken by the project manager. Two levels for the uncertainty proportion are proposed. A low degree of uncertainty proportion corresponds with values that are drawn randomly from an interval with values between $20 \%$ and $40 \%$. A high degree of the uncertainty proportion originates from a random draw with $60 \%$ and $80 \%$ as its lower and upper bound, respectively. The third component of the delays is the size of the delays. This is drawn from a triangular distribution and varies from 1 to 9 , with the mode equal to 4 . The parameter values for the uncertainty threshold 
are equal to 0 and 1.0. Hence, the project will be judged to be highly or only slightly uncertain, respectively. In the remainder of this manuscript, the term uncertainty will be used for the proportion of delays, unless noted otherwise.

\subsection{Results}

In the previous section, the settings for the baseline scenario were discussed. This scenario will now be used to analyze the different links between complexity, uncertainty and how these dimensions are judged. The results for the baseline scenario are divided into 3 paragraphs. The first paragraph deals with the performance of both solution strategies when complexity and uncertainty are assessed correctly. Two situations may occur, namely when the complexity or uncertainty is low and when the complexity or uncertainty is high. The second paragraph takes a look at the two possible judgement errors. One of the dimensions, complexity or uncertainty, may be low in reality but can be judged high. Alternatively, the real complexity or uncertainty may be high but judged to be low. The significance results of a correct assessment and the judgement errors can be found in Tables IX and X. Table IX deals with the significance results of the main experiment, whereas the judgement error results can be found in Table X. The third paragraph looks at how a higher level of effort impacts the cost performance of both solution strategies.

Performance In this paragraph, we limit ourselves to situations in which the decision maker judged the complexity and uncertainty correctly. The following five observations with regard to the performance of both solution strategies can be made:

1. The penalty costs for the time-based strategy $\left(\mathrm{SS}_{1}\right)$ are lower compared to those of the cost-based strategy $\left(\mathrm{SS}_{2}\right)$ across all complexity, uncertainty and penalty levels.

This implies that the project duration attained by $\mathrm{SS}_{1}$ lies closer to the deadline than it is for $\mathrm{SS}_{2}$, resulting in a lower amount of incurred penalty costs.

2. For a high penalty setting, a larger deadline deviation leads to a steep cost deterioration.

It is no surprise that due to this increased importance of the timing aspect, $\mathrm{SS}_{1}$ thrives in a high penalty setting.

3. Even though $\mathrm{SS}_{1}$ has a smaller share of penalty costs, the activity costs of $\mathrm{SS}_{2}$ are much lower, indicating that a better trade-off selection takes place.

The timing aspect does not have a substantial impact when the penalty is low. Hence, $\mathrm{SS}_{2}$ almost always returns better results than $\mathrm{SS}_{1}$. The difference between both strategies is more pronounced for a high degree of complexity.

4. The complex search process for better trade-offs proves advantageous for a cost-based approach $\left(\mathrm{SS}_{2}\right)$. 
When the complexity is high, $\mathrm{SS}_{2}$ is slightly but not significantly better even when the penalty is high. In that case, the proportion of penalty costs is larger than for $\mathrm{SS}_{1}$ but the activity costs are much lower.

5. When there is little uncertainty, $\mathrm{SS}_{2}$ performs better or there is only a small difference compared to $\mathrm{SS}_{1}$. Clearly, a low degree of uncertainty only has a minor impact on a project's duration.

\section{INSERT TABLE IX HERE}

Judgement Error A central topic in this paper is the discrepancy between the real complexity or uncertainty and how it is judged. Hence, judgement errors can be made in which a dimension is low but judged to be high or vice versa. The results of these judgement errors lead to the following three conclusions:

1. A general conclusion for both strategies is that safety is the best policy.

It is better to prepare for the worst and judge the complexity dimension to be worse (highly complex) than it may be in reality (low complexity).

2. For a high penalty setting, the time-based approach $\left(\mathrm{SS}_{1}\right)$ performs well compared to the cost-based $\operatorname{approach}\left(\mathrm{SS}_{2}\right)$.

The preferred solution strategy depends on the (actual and perceived) complexity of the judgement error. Even though the time-based strategy $\left(\mathrm{SS}_{1}\right)$ performs slightly better than the cost-based strategy $\left(\mathrm{SS}_{2}\right)$ when the complexity is high but judged to be low, the difference was found to be statistically insignificant. When the complexity is low but judged to be high, the cost-based strategy comes out on top.

When it comes to the uncertainty dimension, $\mathrm{SS}_{1}$ performs slightly better than $\mathrm{SS}_{2}$ for both judgement error positions. Interestingly, the activity costs are lower for $\mathrm{SS}_{2}$ but the higher penalty costs push the global cost deviation of $\mathrm{SS}_{2}$ higher than that of $\mathrm{SS}_{1}$.

3. For a low penalty setting, the cost-based strategy $\left(\mathrm{SS}_{2}\right)$ clearly outperforms the time-based strategy $\left(\mathrm{SS}_{1}\right)$.

\section{INSERT TABLE X HERE}

Influence of the Level Of Effort In order to ensure that no large differences in the level of effort materialize for the generated projects, the level of effort was controlled using focus and intensity. The focus and intensity settings for the baseline scenario were described in section 5.2. In this section, the effect of an increased level of effort is studied. Three separate experiments were conducted to study the effect of an increased level of effort on the performance of the solution strategies. The global cost deviations of the three experiments can be found in Table A7 of the Appendix. The main findings for each experiment can be summarized as follows:

1. Experiment 1 adopted a focus of $100 \%$ in absence of any uncertainty $(U=0)$. 
The intensity was varied from 0.6 to 1.0 in steps of 0.1 . The results indicate that an increased intensity leads to better cost deviations.

2. Experiment 2 adopted an intensity of $100 \%$ in absence of any uncertainty $(U=0)$.

The focus was varied from 0.6 to 1.0 in steps of 0.1 . Similar to the first experiment, the global cost deviation decreased as the focus was increased, but the decrease was less steep compared to the findings of the first experiment.

3. Experiment 3 reintroduced the uncertainty settings of the baseline scenario, while the focus was kept at $100 \%$ and the intensity was varied again from 0.6 to 1.0 .

Hence, compared to the first experiment, these settings allowed us to explore the influence of the uncertainty. As the intensity (and thus the level of effort) increased, the global cost deviation decreased. However, the cost deviations are higher than those of the first experiment, which can be attributed to uncertainty affecting the activity durations.

Finally, we have also tested the influence of the deadline and the SP level. The deadline parameter was varied by selecting the 25 th and 75 th percentile. Both solution strategies share a decreasing deadline deviation trend as the deadline increases, without leading to different conclusions for the overall performance. The SP factor was varied from 0.1 to 0.9 with steps of 0.1 . No consistent trend for the solution strategies across the complexity and uncertainty dimensions could be established.

\section{Discussion and conclusion}

In this paper, three contributions were made. First, the decisions of students throughout the Project Scheduling Game were translated into two major solution strategies. These are comprised of five building blocks, namely focus, activity criticality, ranking, intensity and action. The first solution strategy focuses on time and employs three mechanisms to approach the deadline. The Greatest Rank Positional weight priority rule is used, as well as a buffer based on the slack duration ratio of Hazir et al. (2010) and a final check to protect the deadline is performed. The second solution strategy heavily focuses on costs, at the expense of an increased exposure to risk. The average most expensive priority rule is used to rank activities. Elitism is applied to only accept cost improvements and non-critical activities' slack is consumed to a larger degree.

Second, complexity and uncertainty were included as contextual factors. The literature overview of section 1 indicated that these are dominant themes and that a link between complexity and project outcome (Hanisch and Wald (2011)) and a continued study of uncertainty (Hall (2012)) were among the challenges for future research. To that end, we have conducted a large computational experiment that allows us to quantify the impact of 
complexity (Maylor et al. (2008)) and uncertainty. The following five conclusions can be drawn from the experiment:

1. A high degree of complexity has a negative effect on the cost deviation.

Since heuristics are designed to make a trade-off between effort and accuracy (Gigerenzer and Gaissmaier (2011)), complex situations call for either more advanced solution methods or for an increase in additional resources and managerial attention, as established by Shenhar (2001).

2. The effect of uncertainty greatly depends on its impact.

Uncertainty was defined as a variation in the duration of an activity. Since most variations led to activity delays, a higher degree of uncertainty generally leads to a longer project duration. Hence, a high degree of uncertainty combined with a severe penalty for deadline overruns led to steep cost increases.

3. Individuals vary in how they assess complexity and uncertainty.

This variation can be partly due to the experience or capability of the project managers in assessing those contextual factors. In the experiment, this was addressed by means of a threshold function, in which the actual and perceived level of complexity and uncertainty were varied. A discrepancy between the actual and perceived level of complexity or uncertainty led to a judgment error. We came to the conclusion that the direction of judgement errors is crucial. Perceiving a project as highly complex and uncertain while this is not true in reality yields significant advantages compared to the opposite scenario. Hence, we recommend project managers who are incapable of correctly assessing a project's complexity and/or uncertainty (e.g. through limited information) to err on the safe side.

4. We identified the conditions in which each solution strategy thrives.

The time-based solution strategy performs particularly well when deadline overruns are heavily penalized and in highly uncertain environments. The cost-based solution strategy yields better results in low penalty and highly complex environments.

5. Increasing the level of effort exhibits a positive effect on the capability of the strategies.

In this paper, we focused on quantifying the effect of complexity and uncertainty on cost outcomes. Hence, the limitation of this paper is that little attention was paid to the behavioural and psychological aspects of complexity and uncertainty. For instance, one can wonder what the effect of tight deadlines on team motivation is and how this relates to previous research on this topic (Chang et al. (2003), Engwall and Westling (2004)). Additionally, demographic variables such as age, background and project role (cf. Ojiako et al. (2014)) could be included, especially when dealing with complexity and uncertainty judgements.

From a data analysis and model perspective, two future research avenues can be identified. First of all, while we 
provided an initial analysis to discern between two major solution strategies, it would be interesting to find out if participants of the PSG switch between strategies throughout the game and by which circumstances this switch is prompted. A similar question arises for niche strategies. Secondly, additional mechanisms can be put in place that further complicate the decision-making process. For instance, increasing or decreasing the time participants have to make decisions throughout the PSG, as well as the presence of a contingency budget may well lead to different choices. A new round of data collection and modeling should be undertaken to bring this meritorious extension to fruition.

\section{Acknowledgements}

We are grateful for the comments made by the editor and referees throughout the review process. Furthermore, we acknowledge the support given by the "Fonds voor Wetenschappelijk Onderzoek (FWO)" under contract number G/0095.10N. 


\section{References}

Azaron, A., Perkgoz, C., and Sakawa, M. (2005). A genetic algorithm approach for the time-cost trade-off in PERT networks. Applied Mathematics and Computation, 168:1317-1339.

Azaron, A. and Tavakkoli-Moghaddam, R. (2007). Multi-objective time-cost trade-off in dynamic PERT networks using an interactive approach. European Journal of Operational Research, 180:1186-1200.

Bein, W., Kamburowski, J., and Stallmann, M. (1992). Optimal reduction of two- terminal directed acyclic graphs. Siam Journal on Computing, 21:1112-1129.

Bourgeois, L. (1985). Strategic goals, perceived uncertainty, and economic performance in volatile environments. Academy of Management Journal, 28: 548-573.

Chang, A. Bordia, P. and Duck, J. (2003). Punctuated equilibrium and linear progression: toward a new understanding of group development. Academy of Management Journal, 46: 106-117.

Cohen, I., Golany, B., and Shtub, A. (2007). The stochastic time-cost tradeoff problem: a robust optimization approach. Networks, 49:175-188.

Crawford, L., Pollack, J., and England, D. (2006). Uncovering the trends in project management: Journal emphases over the last 10 years. International Journal of Project Management, 24:175-184.

De, P., Dunne, E., Ghosh, J., and Wells, C. (1995). The discrete time-cost tradeoff problem revisited. European Journal of Operational Research, 81:225-238.

De, P., Dunne, E., Ghosh, J., and Wells, C. (1997). Complexity of the discrete time/cost trade-off problem for project networks. Operations Research, 45:302-306.

De Reyck, B. and Herroelen, W. (1996). On the use of the complexity index as a measure of complexity in activity networks. European Journal of Operational Research, 91:347- 366.

Demeulemeester, E., De Reyck, B., Foubert, B., Herroelen, W., and Vanhoucke, M. (1998). New computational results on the discrete time/cost trade-off problem in project networks. Journal of the Operational Research Society, 49:1153-1163.

Demeulemeester, E., Elmaghraby, S., and Herroelen, W. (1996). Optimal procedures for the discrete time/cost trade-off problem in project networks. European Journal of Operational Research, 88:50-68.

Dvir, D., Sadeh, A., and Malach-Pines, A. (2006). Projects and project managers: the relationship between project manager's personality, project, project types, and project success. Project Management Journal, $37(5): 36-48$.

Elmaghraby, S. (1977). Activity networks: Project planning and control by network models. New York: John Wiley and Sons, Inc.

Engwall, M. and Westling, G. (2004). Peripety in an R\&D drama: capturing a turnaround in project dynamics. Organization Studies, 25: 1557-1578.

Fang, C. and Marle, F. (2012). A simulation-based risk network model for decision support in project risk management. Decision Support Systems, 52:635-644.

Faria, A. (2001). The changing nature of business simulation/gaming research: A brief history. Simulation \& Gaming, 32:97-110.

Geraldi, J. and Adlbrecht, G. (2007). On faith, fact and interaction in projects. Project Management Journal, 38(1):32-43.

Geraldi, J., Maylor, H., and Williams, T. (2011). Now, let's make it really complex (com- plicated). International Journal of Operations \& Production Management, 31(9):966- 990. 
Ghoddousi, P., Eshtehardian, E., Jooybanpour, S., and Javanmardi, A. (2013). Multi- mode resource-constrained discre time-cost-resource optimization in project scheduling using non-dominated sorting genetic algorithm. Automation in Construction, 30:216- 227.

Gigerenzer, G. and Gaissmaier, W. (2011). Heuristic Decision Making. Annual Review of Psychology, 62: 451482 .

Green, G. (2004). The impact of cognitive complexity on project leadership performance. Information and Software Technology, 46:165-172.

Haapasalo, H. and Hyvönen, J. (2001). Simulating business and operations manage- ment - a learning environment for the electronics industry. International Journal of Production Economics, 73:261-272.

Hall, N. (2012). Project management: recent developments and research opportunities. Journal of Systems Science and Systems Engineering, 21(2):129-143.

Hanisch, B. and Wald, A. (2011). A project management research framework integrating multiple theoretical perspectives and influencing factors. Project Management Journal, 42(3):4-22.

Hazir, O., Erel, E., and G“unalay, Y. (2011). Robust optimization models for the discrete time/cost trade-off problem. International Journal of Production Economics, 130:87-95.

Hazir, O., Haouari, M., and Erel, E. (2010). Robust scheduling and robustness measures for the discrete time/cost trade-off problem. European Journal of Operations Research, 207:633-643.

Hindelang, T. and Muth, j. (1979). A dynamic programming algorithm for decision CPM networks. Operations Research, 27:225-241.

Howell, D., Windahl, C., and Seidel, R. (2010). A project contingency framework based on uncertainty and its consequences. International Journal of Project Management, 28:256-264.

Jaafari, A. (2003). Project management in the age of complexity and change. Project Management Journal, 34(4):47-57.

Ke, H., Ma, W., and Ni, Y. (2009). Optimization models and a GA-based algorithm for stochastic time-cost trade-off problem. Applied Mathematics and Computation, 215:308-313.

Ke, H., Ma, W. and Ni, Y. (2012). Modelling stochastic project time-cost trade-offs with time-dependent activity durations. Applied Mathematics and Computation, 218:9462-9469.

Kelley, J. (1961). Critical path planning and scheduling: Mathematical basis. Operations Research, 9:296-320.

Kelley, J. and Walker, M. (1959). Critical path planning and scheduling: An introduction. Mauchly Associates, Ambler, PA.

Klerides, E. and Hadjiconstantinou, E. (2010). A decomposition-based stochastic programming approach for the project scheduling problem under time/cost trade-off settings and uncertain durations. Computers \& Operations Research, 37:2131-2140.

Kolb, D. (1984). Experiential Learning: Experience as The Source of Learning and Development. Prentice-Hall. Mastor, A. (1970). An experimental and comparative evaluation of production line balancing techniques.

Management Science, 16:728-746.

Maylor, H., Vidgen, R., and Carver, S. (2008). Managerial complexity in project-based operations: a grounded model and its implications for practice. Project Management Journal, 39:S15-S26.

Meyer, W. and Shaffer, L. (1965). Extending CPM for multiform project time-cost curves. Journal of the Construction Division, Proceedings of the ASCE, 91:45-65.

Mintzberg, H., Raisinghani, D. and Theoret, A. (1976). The structure of unstructured decision processes. Administrative Science Quarterly, 21: 246-275. 
Moder, J., Phillips, C., and Davis, E. (1983). Project Management with CPM, PERT and Precedence Diagramming. Nostrand Reinhold, New York.

Mokhtari, H., Kazemzadeh, R., and Salmasnia, A. (2011). Time-cost tradeoff analysis in project management: an ant system approach. IEEE Transactions on Engineering Management, 58:36-43.

Müller, R. and Turner, J. (2007). Matching the project manager's leadership style to project type. International Journal of Project Management, 25(1):21-32.

Nutt, P. (1998). How decision makers evaluate alternative and the influence of complexity. Management Science, 44(8):1148-1166.

Ojiako, U., Chipulu, M., Gardiner, P., Williams, T., Mota, C., Maguire, S., Shou, Y., and Stamati, T. (2014).

Effect of project role, age and gender differences on the formation and revision of project decision judgements. International Journal of Project Management, 32:556-567.

Osman, M. (2010). Controlling uncertainty: a review of human behaviour in complex dynamic environments. Psychological Bulletin, 136(1):65-86.

Parente, D., Stephan, J., and Brown, R. (2012). Facilitating the acquisition of strategic skills: the role of traditional and soft managerial skills. Management Research Review, 35:1004-1028.

Pascoe, T. (1966). Allocation of resources - CPM. Revue Fran , caise de Recherche Op 'erationnelle, 38:31-38. Pich, M., Loch, C., and De Meyer, A. (2002). On uncertainty, ambiguity, and complexity in project management. Management Science, 48:1008-1023.

Phillips, S. and Dessouky, M. (1977). Solving the project time/cost tradeoff problem using the minimal cut concept. Management Science, 24:393-400.

Pollack, J. (2007). The changing paradigms of project management. International Journal of Project Management, 25:266-274.

Pour, N., Modarres, M., Aryanejad, M., and Moghadam, R. (2010). The discrete time-cost-quality trade-off problem using a novel hybrid genetic algorithm. Applied Mathematical Sciences, 42:2081-2094.

Robinson, D. (1975). A dynamic programming solution to cost/time trade-off for CPM. Management Science, 22:158-166.

Saunders, P. (1997). Experiential learning, cases, and simulations in business communication. Business Communication Quarterly, 60:97-114.

Schumann, P., Anderson, P., and Scott, T. (1997). Using computer-based simulation exercises to teach business ethics. Teaching Business Ethics, 1:163-181.

Shenhar, A. (2001). One size does not fit all projects: exploring classical contingency domains. Management Science, 47(3):394-414.

Söderlund, J. (2011). Pluralism in project management: Navigating the crossroads of specialization and fragmentation. International Journal of Management Reviews, 13:153-176.

Sommer, S. and Loch, C. (2004). Selectionism and learning in projects with complexity and unforeseeable uncertainty. Management Science, 50:1334-1347.

Sonmez, R. and Bettemir, O. (2012). A hybrid genetic algorithm for the discrete time-cost trade-off problem. Expert Systems with Applications, 39:11428-11434.

Stumpf, S., Dunbar, R., and Mullen, T. (1991). Developing entrepreneurial skills through the use of behavioural simulations. Journal of Management Development, 10:32-45.

Tareghian, H. and Taheri, S. (2006). On the discrete time, cost and quality trade-off problem. Applied 
Tareghian, H. and Taheri, S. (2007). A solution procedure for the discrete time, cost and quality tradeoff problem using electromagnetic scatter search. Applied Mathematics and Computation, 190:1136-1145.

Tavares, L. (1999). Advanced models for project management. Kluwer Academic Publishers, Dordrecht, 1999. Van Ackere, A., Larsen, E., and Morecroft, J. (1993). Systems thinking and business process redesign: an application to the beer game. European Management Journal, 11:412-423.

Thomas, J. and Mengel, T. (2008). Preparing project managers to deal with complexity - Advanced project management education. International Journal of Project Management, 26:304-315.

Turner, J. and Mu “1ler, R. (2005). The project manager's leadership style as a success factor on projects: a literature review. Project Management Journal, 36(1):49-61.

Vanhoucke, M. (2010). Using activity sensitivity and network topology information to monitor project time performance. Omega The International Journal of Management Science, 38:359-370.

Vanhoucke, M., Coelho, J., Debels, D., Maenhout, B., and Tavares, L. (2008). An evaluation of the adequacy of project network generators with systematically sampled networks. European Journal of Operational Research, $187: 511-524$

Vanhoucke, M. and Debels, D. (2007). The discrete time/cost trade-off problem: Extensions and heuristic procedures. Journal of Scheduling, 10:311-326.

Vanhoucke, M., Demeulemeester, E., and Herroelen, W. (2002). Discrete time/cost trade-offs in project scheduling with time-switch constraints. Journal of the Operational Research Society, 53:741-751.

Vanhoucke, M., Vereecke, A., and Gemmel, P. (2005). The project scheduling game (PSG): Simulating time/cost trade-offs in projects. Project Management Journal, 51:51-59.

Vidal, L.-A. and Marle, F. (2008). Understanding project complexity: implications on project management. Kybernetes, 37(8):1094-1110.

Walker, M. and Sawyer, J. (1959). Project Planning and Scheduling. Technical Report Report 6959, E.I. duPont de Nemours and Co., Wilmington, Delaware.

Ward, S. and Chapman, C. (2003). Transforming project risk management into project uncertainty management. International Journal of Project Management, 21:97-105.

Wolfe, J. (1997). The effectiveness of business games in strategic management course work. Simulation \& Gaming, 28:360-376.

Yang, L.-R., Huang, C.-F., and Wu, K.-S. (2011). The association among project man- ager's leadership style, teamwork and project success. International Journal of Project Management, 29:258-267. 


\section{Figures}

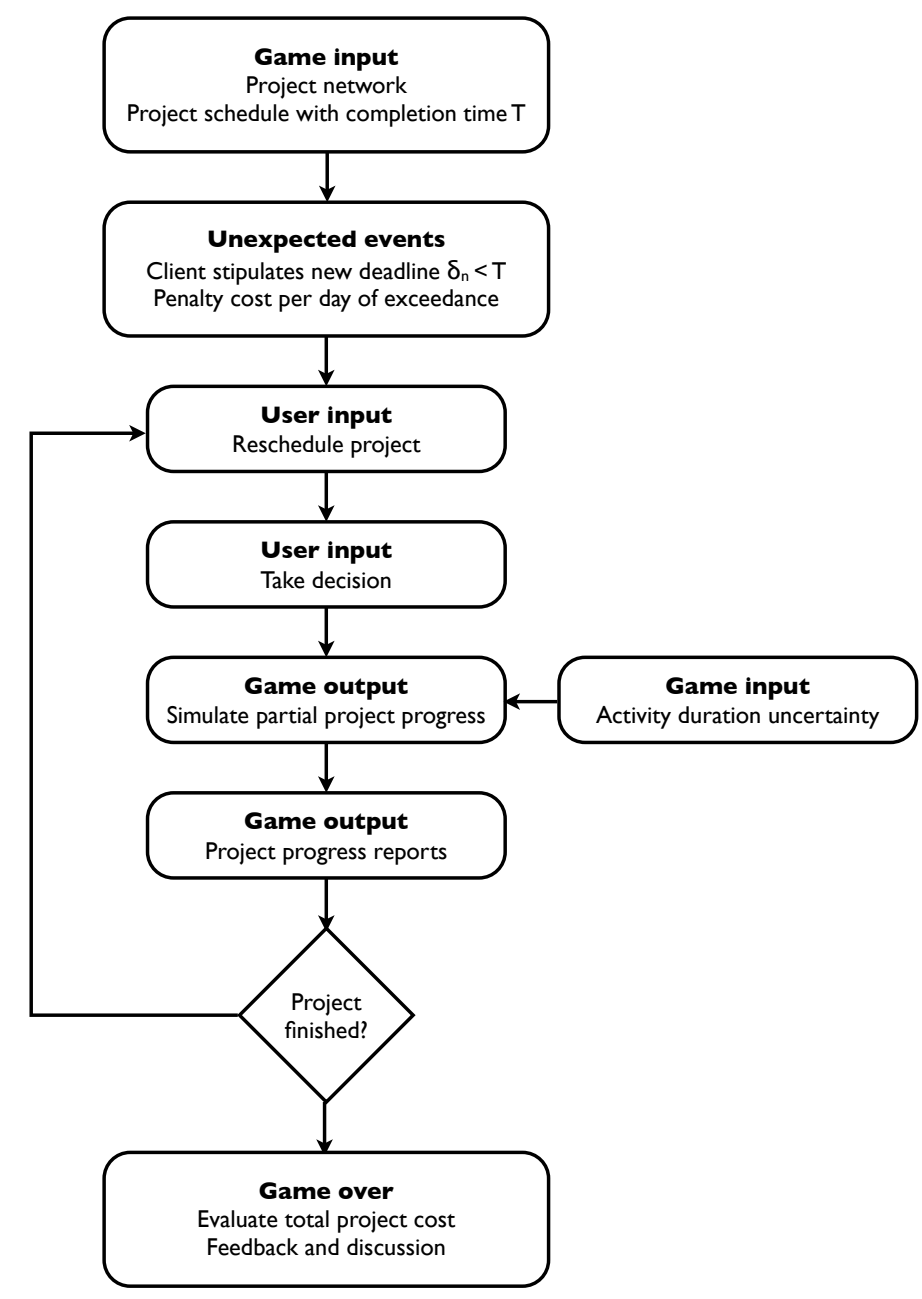

Figure I: The process of the Project Scheduling Game (source: PM Knowledge Center (www.pmknowledgecenter.com)) 


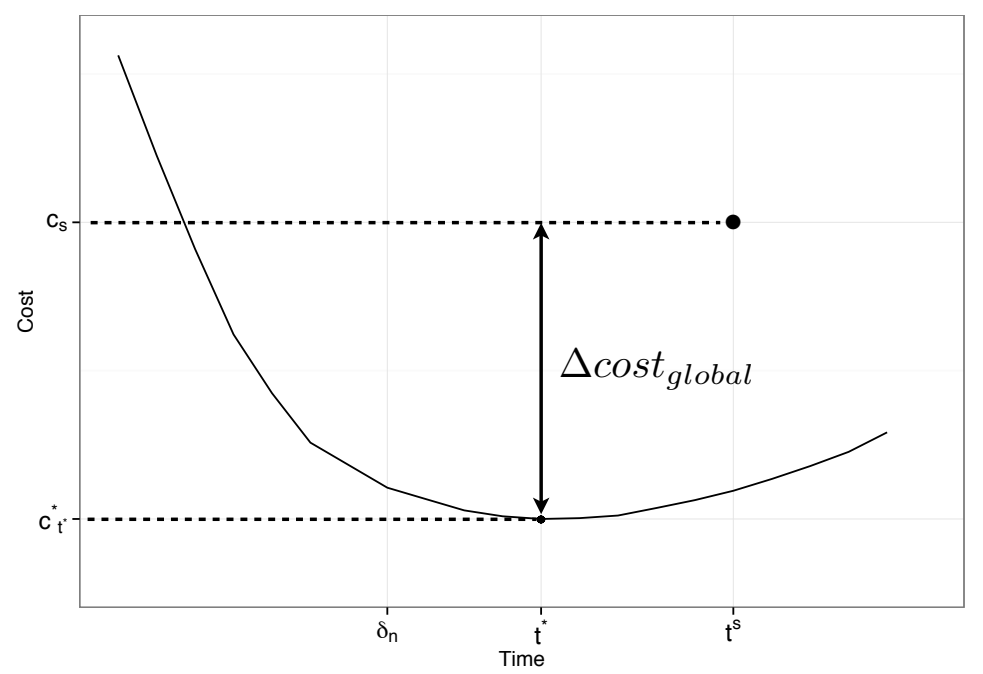

Figure II Visualization of the global cost deviation

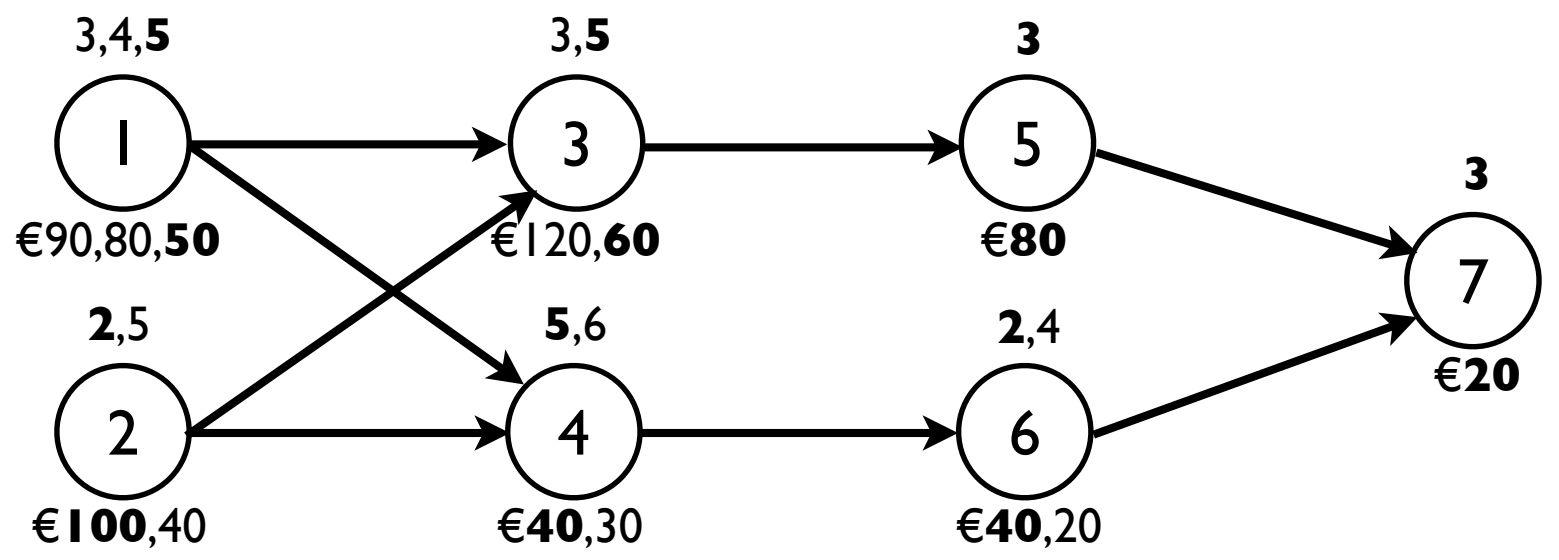

Figure IIIa Activity on the Node (AoN) notation of the illustrative example

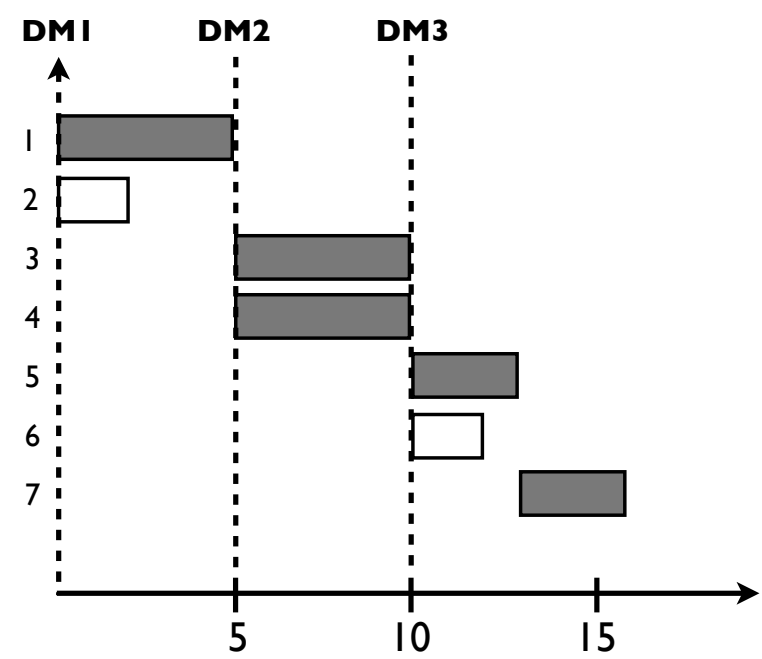

Figure IIIb Gantt-chart of the illustrative example 


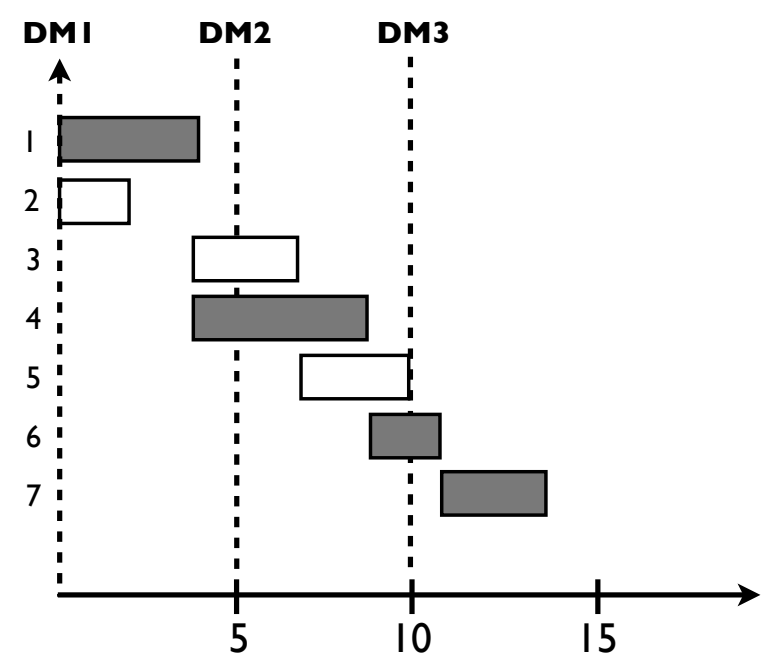

Figure IV New Gantt-chart after actions on activities 3 and 1

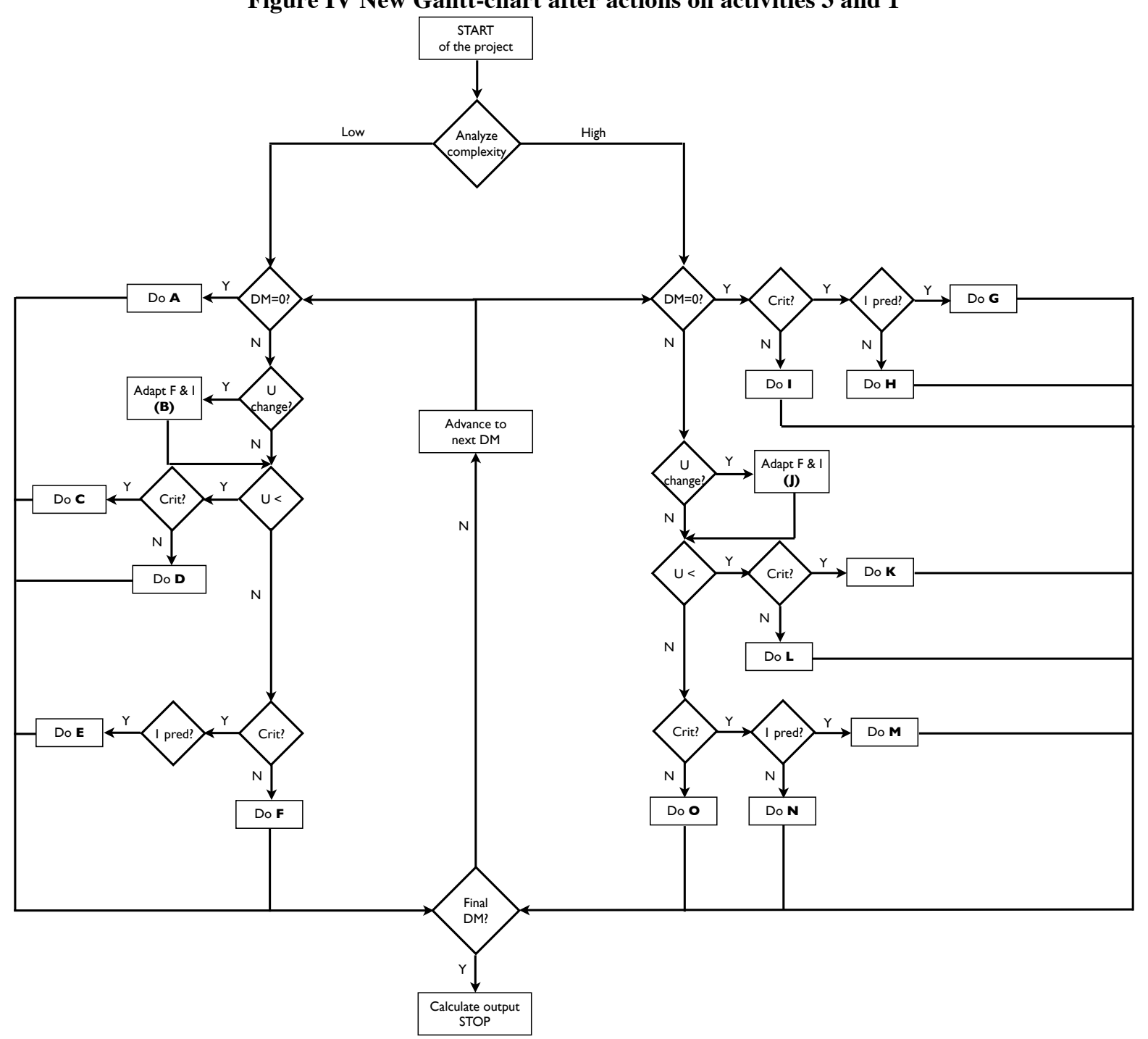

Figure V: Overview of the framework of the solution strategies 


\section{Tables}

Table I: Overview of current literature on the Discrete Time/Cost Trade-off Problem

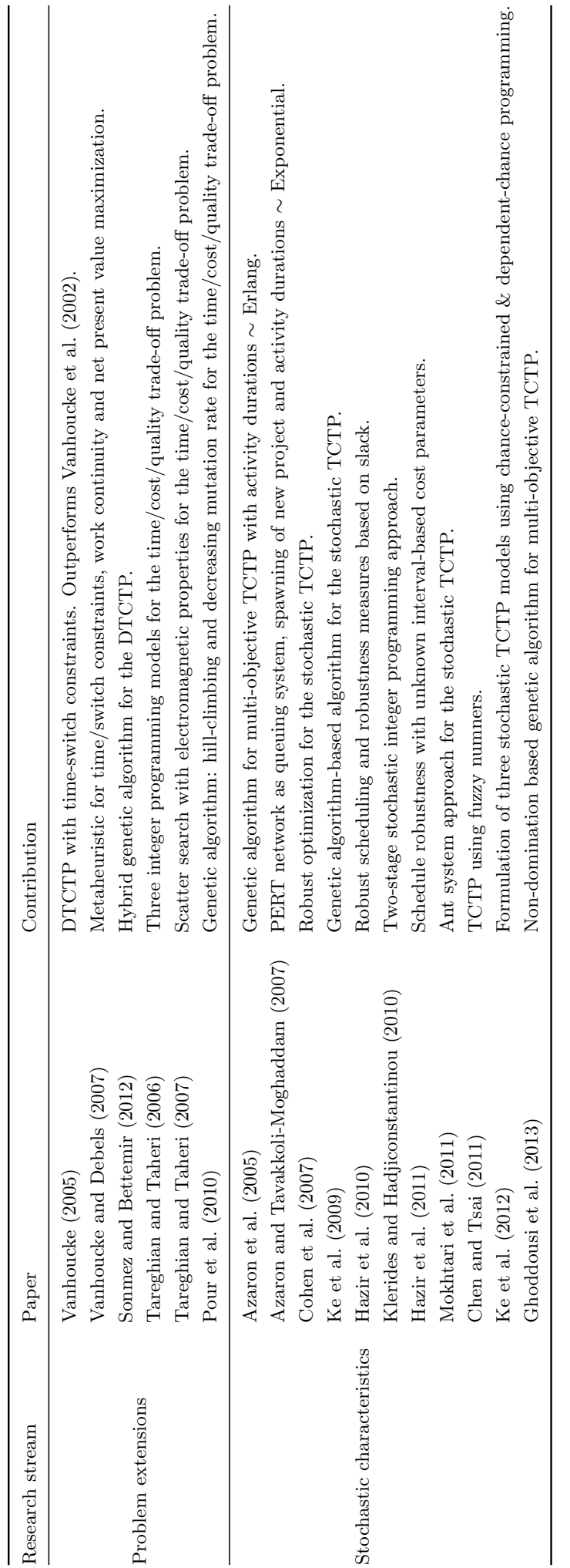


Table II: Overview of the student results

\begin{tabular}{lccccr}
\hline \multirow{2}{*}{ Student group } & \multirow{2}{*}{ \#students } & \multicolumn{4}{c}{ Cost deviation (\%) } \\
& & Minimum & Maximum & Average & $\sigma$ \\
\hline Business Engineering (UGent) & 176 & 0.19 & 12.46 & 1.52 & 1.76 \\
Civil Engineering (UGent) & 203 & 0.24 & 12.76 & 1.29 & 1.44 \\
Civil Engineering (UCL) & 36 & 0.38 & 4.52 & 1.75 & 0.82 \\
Managmeent Science (UCL) & 5 & 0.50 & 1.79 & 1.10 & 0.54 \\
Management (VBS \& EDHEC) & 24 & 0.38 & 4.60 & 1.72 & 1.34 \\
\hline
\end{tabular}

Table III: Overview of the type of actions

\begin{tabular}{ll}
\hline Type of action & Description \\
\hline Swap & Select neighbouring trade-off. \\
Slack consumption & increase duration until no slack is left. \\
Minimum cost slope & Select trade-off with maximum duration decrease at minimum cost. \\
Maximum revenue slope & Select trade-off with minimum duration increase at maximum savings. \\
Enumeration & Enumerate all trade-off for set of activities. \\
Protect deadline & Decrease/increase project duration until acceptable deviation from $\delta_{n}$. \\
\hline
\end{tabular}

Table IV: Overview of the 5 components for the illustrative example

\begin{tabular}{ll}
\hline Component & Setting \\
\hline Focus & 0.67 \\
Activity criticality & Critical \\
Ranking & Most Expensive Activity \\
Intensity & 0.67 \\
Action & Crash with swap move \\
\hline Parameter & Value \\
\hline Deadline & 13 days \\
Penalty & $€ 100$ \\
\hline
\end{tabular}


Table V: overview of the actions for the 3 alternative strategies and their outcome (NA indicates an activity cannot be crashed any more)

\begin{tabular}{|c|c|c|c|c|c|c|}
\hline \multirow{2}{*}{ Strategy } & \multirow{2}{*}{ Decision Moment } & \multirow{2}{*}{ Activity } & \multicolumn{2}{|c|}{ Old trade-off } & \multicolumn{2}{|c|}{ New trade-off } \\
\hline & & & Time & Cost & Time & Cost \\
\hline \multirow{3}{*}{ Strategy 1} & 1 & 1 & 5 & 50 & 4 & 80 \\
\hline & 2 & 5 & 3 & 80 & & NA \\
\hline & 3 & 5 & 3 & 80 & & NA \\
\hline \multirow{5}{*}{ Strategy 2} & \multirow{2}{*}{1} & 3 & 5 & 60 & 3 & 120 \\
\hline & & 1 & 5 & 50 & 4 & 80 \\
\hline & \multirow[t]{2}{*}{2} & 6 & 2 & 40 & & NA \\
\hline & & 6 & 2 & 40 & & $\mathrm{NA}$ \\
\hline & 3 & 7 & 3 & 20 & & $\mathrm{NA}$ \\
\hline \multirow{11}{*}{ Strategy 3} & \multirow{6}{*}{1} & 5 & 3 & 80 & & $\mathrm{NA}$ \\
\hline & & 3 & 5 & 60 & 3 & 120 \\
\hline & & 1 & 5 & 50 & 4 & 80 \\
\hline & & 6 & 2 & 40 & & NA \\
\hline & & 4 & 5 & 40 & & $\mathrm{NA}$ \\
\hline & & 7 & 3 & 20 & & NA \\
\hline & \multirow{3}{*}{2} & 4 & 5 & 40 & & $\mathrm{NA}$ \\
\hline & & 6 & 2 & 40 & & NA \\
\hline & & 7 & 3 & 20 & & NA \\
\hline & \multirow{2}{*}{3} & 6 & 2 & 40 & & NA \\
\hline & & 7 & 3 & 20 & & NA \\
\hline Strategy & \multicolumn{2}{|c|}{ Project duration } & \multicolumn{2}{|c|}{ Project cost } & Level & Of Effort \\
\hline Do nothing & \multicolumn{2}{|l|}{16} & \multicolumn{2}{|c|}{690} & & 0 \\
\hline Strategy 1 & \multicolumn{2}{|l|}{15} & \multicolumn{2}{|c|}{620} & & 1 \\
\hline Strategy 2 & \multicolumn{2}{|l|}{14} & \multicolumn{2}{|c|}{580} & & 3 \\
\hline Strategy 3 & \multicolumn{2}{|l|}{14} & \multicolumn{2}{|c|}{580} & & 3 \\
\hline
\end{tabular}

Table VI: Principal differences between the solution strategies

Solution strategies

$\mathrm{SS}_{1}$

$\mathrm{SS}_{2}$

GRPW priority rule Avg MEA priority rule

Buffer (SDR of 30\%) Elitism (only accept cost improvement)

Protect deadline Slack consumption 
Table VII: Overview of the solution strategies and their components

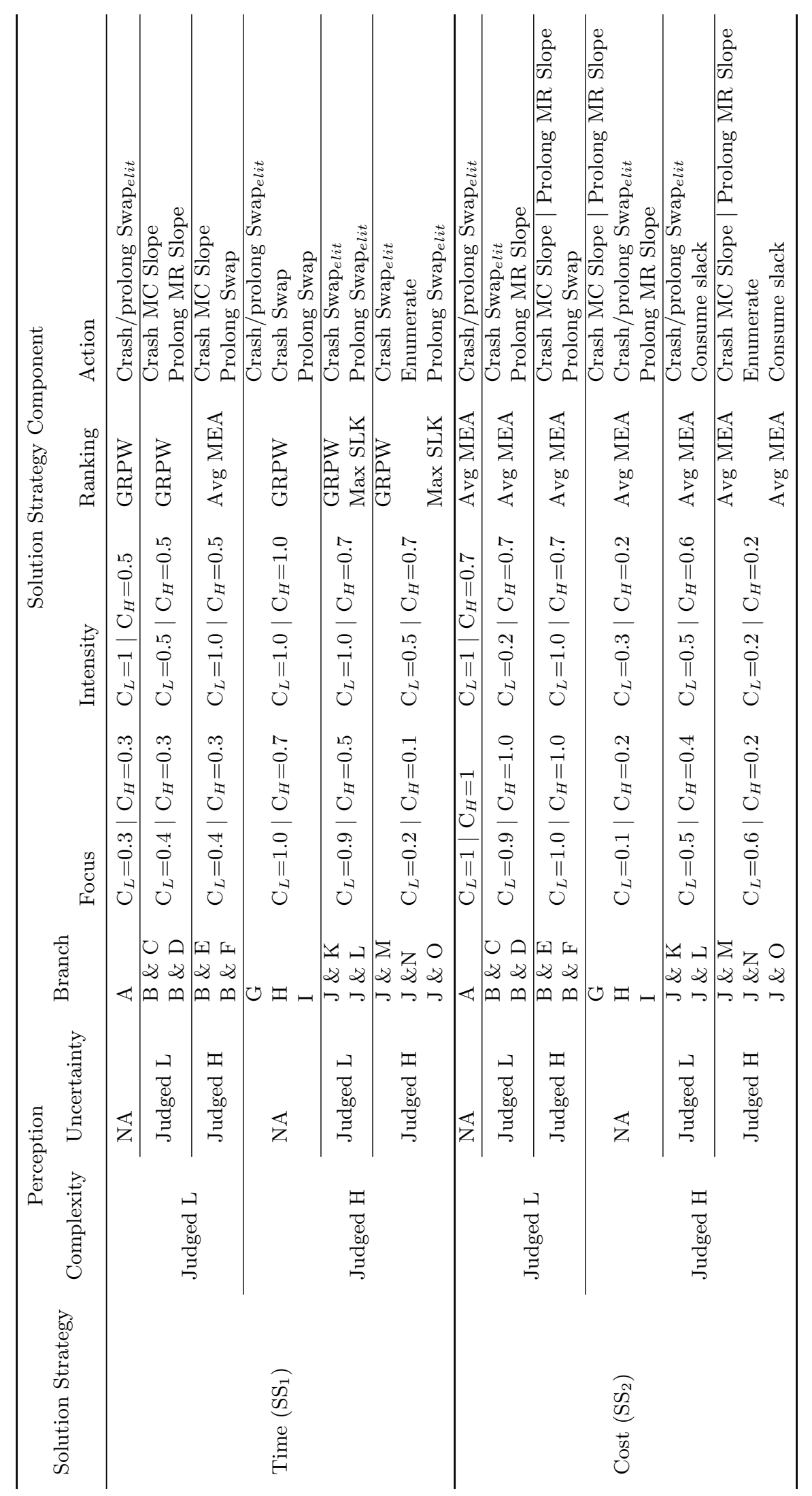


Table VIII: Overview of the data generation parameters

\begin{tabular}{|c|c|c|c|}
\hline \multicolumn{3}{|c|}{ Description } & Settings \\
\hline & \multicolumn{2}{|l|}{ SP-factor } & $0.1-0.9, \Delta=0.1$ \\
\hline & \multicolumn{2}{|l|}{ \#Projects } & 100 \\
\hline & \multicolumn{2}{|l|}{ Trade-offs } & Random \\
\hline Project parameters & \multicolumn{2}{|l|}{$\theta$} & $0.25-0.75, \Delta=0.25$ \\
\hline & \multicolumn{2}{|l|}{ Penalty } & $€ 350-€ 3,500$ \\
\hline & \multicolumn{2}{|c|}{ Activity Costs } & $\sim \mathrm{R}(500-2,500)$ \\
\hline & \multicolumn{2}{|c|}{ Activity Durations } & $\sim \mathrm{R}(10-20)$ \\
\hline \multirow{4}{*}{ Baseline scenario } & \multicolumn{2}{|l|}{ SP-factor } & 0.5 \\
\hline & & 0.5 \\
\hline & \multirow{2}{*}{\multicolumn{2}{|c|}{ Penalty }} & Low: $€ 350$ \\
\hline & & & High: $€ 3,500$ \\
\hline \multirow{6}{*}{ Complexity \& Uncertainty } & \multirow{3}{*}{ Complexity } & Low & $\sim \operatorname{Tri}(1,4,6)$ \\
\hline & & High & $\sim \operatorname{Tri}(4,7,9)$ \\
\hline & & Thresholds & $0-10$ \\
\hline & \multirow{3}{*}{ Uncertainty } & Low & $\sim \mathrm{R}(0.2-0.4)$ \\
\hline & & High & $\sim \mathrm{R}(0.6-0.8)$ \\
\hline & & Thresholds & $0-1.0$ \\
\hline
\end{tabular}

Table IX: results of the main experiment (correct judgement). An asterisk denotes a significant

difference: $p<0.05$

\begin{tabular}{|c|c|c|c|c|c|c|c|c|}
\hline \multirow{2}{*}{ Dimension } & \multirow{2}{*}{ Actual \& Perceived } & \multirow{2}{*}{ Penalty } & \multicolumn{3}{|c|}{ Global cost deviation } & \multicolumn{3}{|c|}{ Penalty share } \\
\hline & & & $\mathrm{SS}_{1}$ & $\mathrm{SS}_{2}$ & Sign. & $\mathrm{SS}_{1}$ & $\mathrm{SS}_{2}$ & Sign. \\
\hline \multirow{4}{*}{ Complexity } & \multirow{2}{*}{ Low } & Low & $11.65 \%$ & $5.90 \%$ & $*$ & $2.10 \%$ & $5.06 \%$ & $*$ \\
\hline & & High & $20.14 \%$ & $27.32 \%$ & $*$ & $11.47 \%$ & $25.21 \%$ & $*$ \\
\hline & \multirow{2}{*}{ High } & Low & $25.42 \%$ & $13.13 \%$ & $*$ & $1.04 \%$ & $6.30 \%$ & $*$ \\
\hline & & High & $25.61 \%$ & $25.30 \%$ & & $4.82 \%$ & $15.18 \%$ & $*$ \\
\hline \multirow{4}{*}{ Uncertainty } & \multirow{2}{*}{ Low } & Low & $20.61 \%$ & $13.08 \%$ & $*$ & $0.97 \%$ & $3.84 \%$ & $*$ \\
\hline & & High & $21.67 \%$ & $22.55 \%$ & $*$ & $4.56 \%$ & $11.42 \%$ & $*$ \\
\hline & \multirow{2}{*}{ High } & Low & $17.97 \%$ & $11.27 \%$ & $*$ & $1.94 \%$ & $5.08 \%$ & $*$ \\
\hline & & High & $25.75 \%$ & $28.12 \%$ & $*$ & $12.88 \%$ & $21.57 \%$ & $*$ \\
\hline
\end{tabular}

Table X: results of the main experiment (judgement error). An asterisk denotes a significant difference: $p$

$<0.05$

\begin{tabular}{|c|c|c|c|c|c|c|c|c|c|}
\hline \multirow{2}{*}{ Dimension } & \multirow{2}{*}{ Actual } & \multirow{2}{*}{ Perceived } & \multirow{2}{*}{ Penalty } & \multicolumn{3}{|c|}{ Global cost deviation } & \multicolumn{3}{|c|}{ Penalty share } \\
\hline & & & & $\mathrm{SS}_{1}$ & $\mathrm{SS}_{2}$ & Sign. & $\mathrm{SS}_{1}$ & $\mathrm{SS}_{2}$ & Sign. \\
\hline \multirow{4}{*}{ Complexity } & \multirow{2}{*}{ Low } & \multirow{2}{*}{ High } & Low & $13.48 \%$ & $7.79 \%$ & $*$ & $1.88 \%$ & $3.34 \%$ & $*$ \\
\hline & & & High & $20.96 \%$ & $18.84 \%$ & $*$ & $12.41 \%$ & $13.04 \%$ & \\
\hline & \multirow{2}{*}{ High } & \multirow{2}{*}{ Low } & Low & $27.10 \%$ & $21.52 \%$ & $*$ & $1.26 \%$ & $3.41 \%$ & $*$ \\
\hline & & & High & $28.84 \%$ & $29.13 \%$ & & $6.53 \%$ & $11.92 \%$ & $*$ \\
\hline \multirow{4}{*}{ Uncertainty } & \multirow{2}{*}{ Low } & \multirow{2}{*}{ High } & Low & $19.07 \%$ & $11.25 \%$ & $*$ & $1.03 \%$ & $4.27 \%$ & $*$ \\
\hline & & & High & $21.04 \%$ & $21.13 \%$ & & $5.47 \%$ & $12.30 \%$ & $*$ \\
\hline & \multirow{2}{*}{ High } & \multirow{2}{*}{ Low } & Low & $20.01 \%$ & $12.74 \%$ & $*$ & $2.33 \%$ & $4.93 \%$ & $*$ \\
\hline & & & High & $27.09 \%$ & $28.79 \%$ & $*$ & $12.33 \%$ & $20.06 \%$ & $*$ \\
\hline
\end{tabular}




\section{A. Appendix}

This appendix contains results or clarifications that were either too expansive to add to the main text of the manuscript or did not alter the main insights of the paper. It provides an example of the data generation process (section 6.1) and the link with Table 3 of the main text.

\section{A1. Data Generation example}

In this section, the data generation of the computational experiment, found in section 6.1 , is illustrated by means of an example of the dataset. The different steps are outlined below:

- Network Generation: generate a network with 32 activities and a value of the SP indicator equal to 0.8 The Activity-on-the-Node (AoN) representation is given in figure A1.

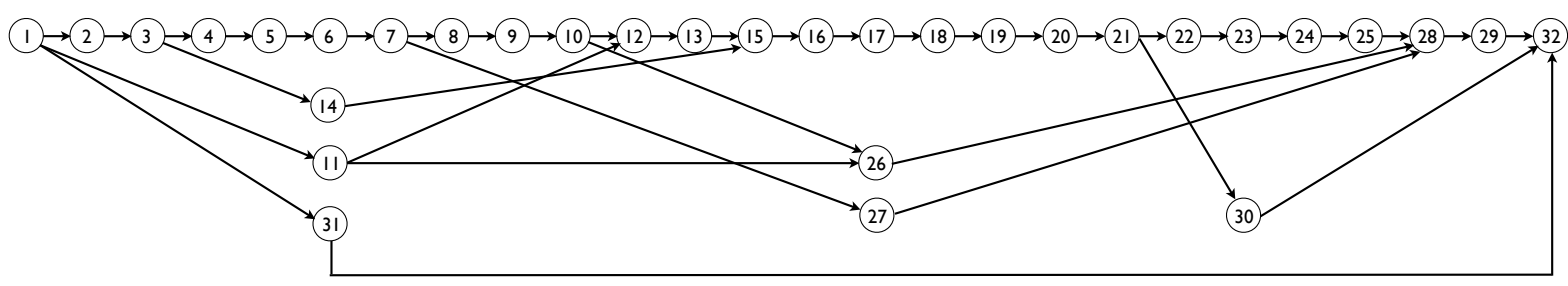

Figure A1: AoN representation of the generated network

- Generate time/cost trade-offs: in this example, a low complexity will be maintained. For each activity, the number of trade-offs is drawn from a triangular distribution with 1,4 and 6 as the minimum, mode and maximum respectively. Each trade-off has a duration between 10 and 20 time units and a cost between 500 and 2,500 monetary units. A full overview of the generated time/cost trade-offs for all activities can be found in Table A1. 
Table A1: Overview of the generated time/cost trade-offs for the data generation example

network

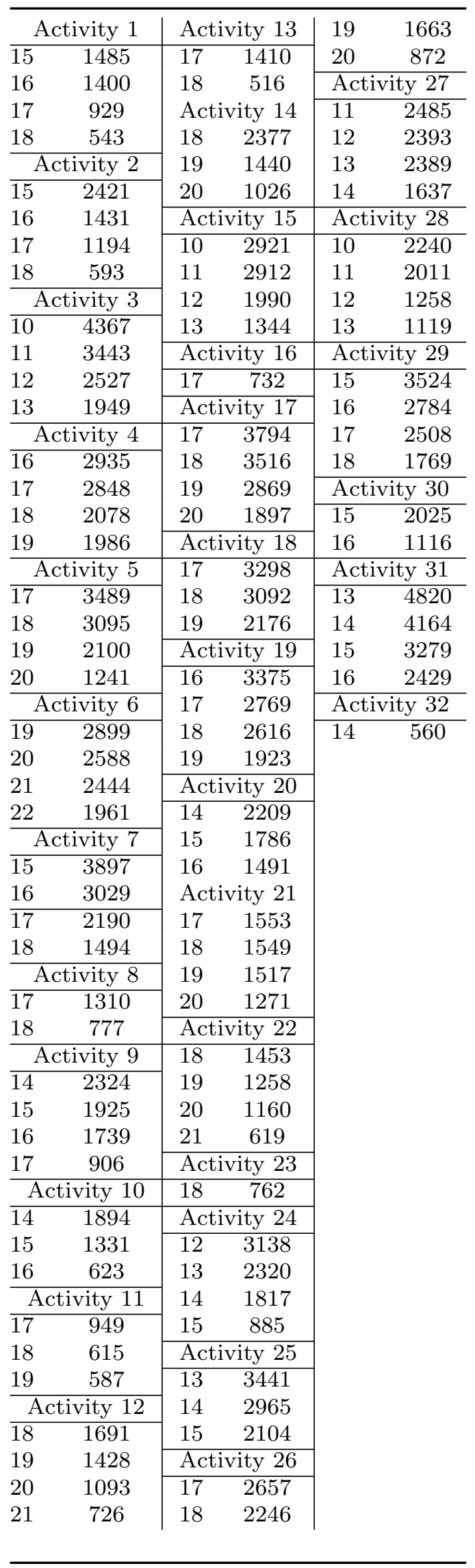


- Generate delays: the uncertainty proportion determines the amount of activities that will be subject to a delay. In this instance, a value of 0.2 is generated, implying that $0.2 * 32 \approx 6$ activities will be delayed. The size of the delays is drawn from a triangular distribution with 1 and 9 as the minimum and maximum and a mode equal to 4 . An overview of the activities that are delayed is shown in Table A2.

\section{Table A2: Overview of the generated delays}

\begin{tabular}{cc}
\hline Activity & Delay \\
\hline 6 & 6 \\
8 & 5 \\
10 & 4 \\
13 & 6 \\
15 & 3 \\
25 & 5 \\
\hline
\end{tabular}

- Generate deadline and penalty: the example is solved exactly, resulting into an efficient time/cost profile. Since no penalty is imposed yet, lengthening the project leads to cost reductions. The deadline is set to 0.5 in this example, which corresponds with the time value of the $20^{\text {th }}(0.5 * 40$ timepoints $)$ point of the efficient time/cost profile. For every day the deadline is exceeded, a penalty cost of 350 monetary units is incurred. Figure A2 shows how the penalty negatively affects the efficient time/cost profile.

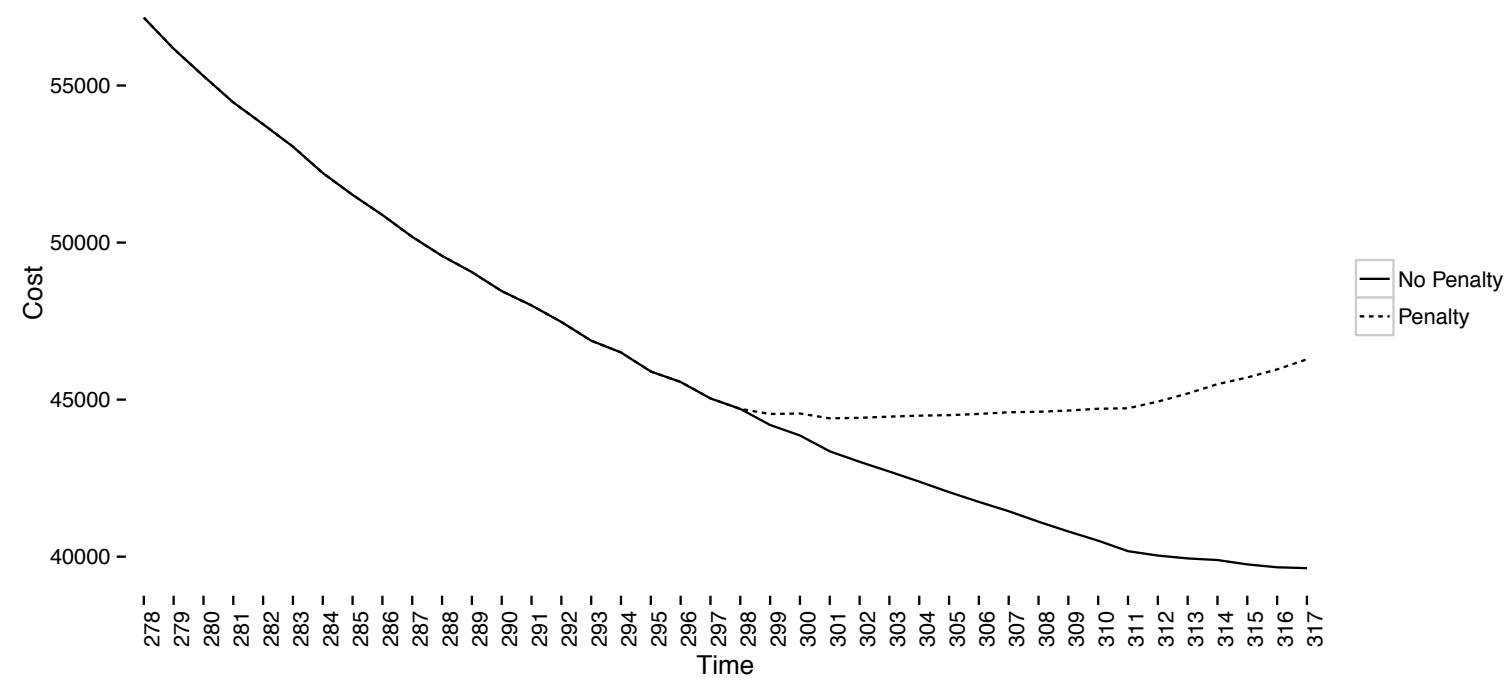

Figure A2: efficient time/cost profile with and without the penalty of 350 monetary units

- Apply one of the solution strategies to the problem at hand. In this example, the actual complexity is low. Assume that the thresholds for complexity and uncertainty are equal to 0 . In that case, complexity and uncertainty will be judged low. This implies that for the time-based strategy, the focus will be equal to 0.4 and the intensity will be 0.5 . The GRPW priority rule will be invoked. Critical activities will be crashed according to the minimum cost slope, whereas non-critical activities will be prolonged 
following the maximum revenue slope. These settings can all be found in Table 3 of the manuscript. The global cost deviation of the time-based solution strategy for this example is equal to $16.12 \%$, with the activity cost making up $100 \%$ of the global cost deviation. Hence, the time-based solution strategy manages to finish before the project's deadline. 